\title{
Dopaminergic modulation of stress-induced alterations in goal-directed behaviour and associated brain activation
}

\author{
Peter van Ruitenbeek ${ }^{1}$, Conny Quaedflieg ${ }^{1}$, Bart Hartogsveld ${ }^{1}$, and Tom Smeets ${ }^{2}$ \\ ${ }^{1}$ Maastricht University \\ ${ }^{2}$ Tilburg University
}

May 5, 2020

\begin{abstract}
Background and purpose: Being exposed to acute stress may cause people to behave more habitual, which purportedly is associated stress-induced increased dopamine release. In contrast, experimental rises in systemic dopamine levels have been shown to increase goal-directed behaviour and, thus, decrease habitual control. Whether experimentally increased dopamine functioning can modulate stress-induced reductions in goal-directed behaviour and its neural substrates, is currently unknown. Experimental approach: To assess whether increased dopamine functioning reduces stress effects on goal-directed behaviour, 100 participants were recruited who were randomly assigned to one of four conditions in a $2 \times 2$ between participants design. Participants underwent a stress induction protocol (Maastricht Acute Stress Test; MAST) or a control procedure and received methylphenidate (40 mg, oral) or placebo. In a well-established instrumental learning paradigm, participants were trained to learn stimulus-response-outcome associations, after which rewards were selectively devalued and participants' goal-directed behaviour was assessed at peak cortisol/methylphenidate concentrations in a magnetic resonance imaging scanner to assess brain activation. Key results: The MAST effectively increased physiological measures of stress (salivary cortisol, blood pressure) and subjective stress. Methylphenidate also increased cortisol levels over time. While stress selectively reduced goal-directed behaviour, this effect was not modulated by methylphenidate. However, methylphenidate modulated stress effects on activation in paracingulate, orbitofrontal cortex, and anterior cingulate associated with expected value representation in goal-directed behaviour. Conclusion and implications: Our neuroimaging data suggest increased dopamine levels reverse stress-induced changes in brain activation associated with goal-directed behaviour. These effects may be relevant for preventing stress-induced relapse in addictive behaviour.
\end{abstract}

\section{Hosted file}

BJP_paper.docx available at https://authorea.com/users/302245/articles/432497-dopaminergicmodulation-of-stress-induced-alterations-in-goal-directed-behaviour-and-associatedbrain-activation 


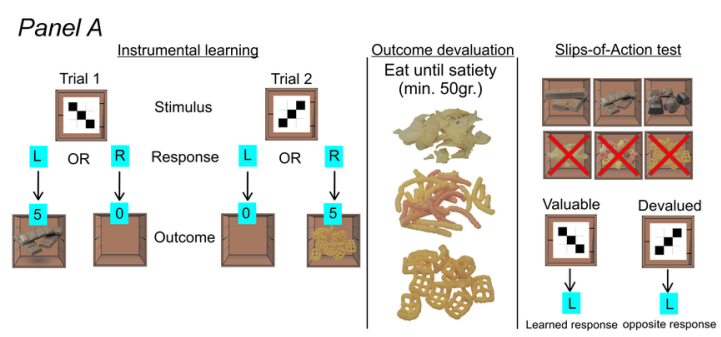

Panel C

Panel B

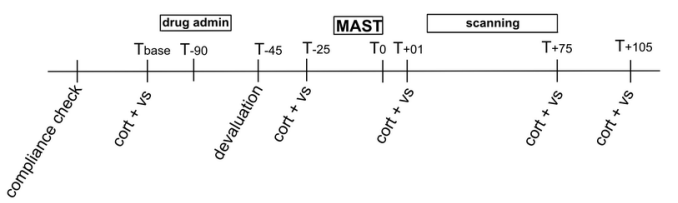

Raw structural data

Brain extraction (BET2)

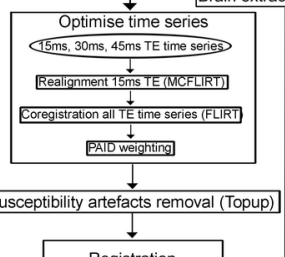

Registration

Co.registration (FLIRT)

Normalisation [FNIRT]

Motion correction (MCFLIRT)

$\frac{\downarrow}{\text { Brain extraction (BET) }}$
$\downarrow$
$\frac{\downarrow}{\text { Smoothing }}$
$\downarrow$
Motion induced artefact reduction (ICA-AROMA)

CSF and WM signal extraction

High pass filtering
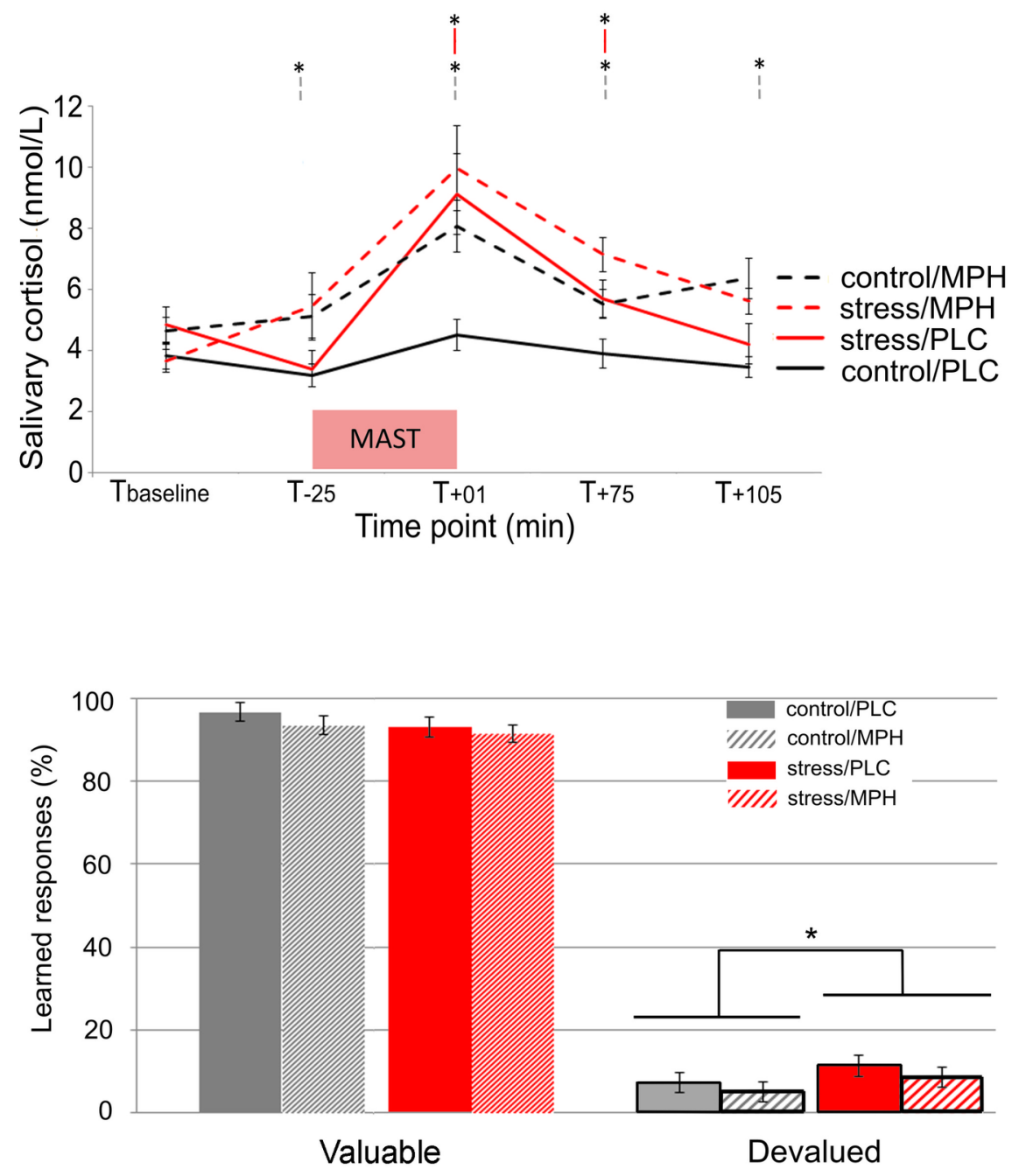


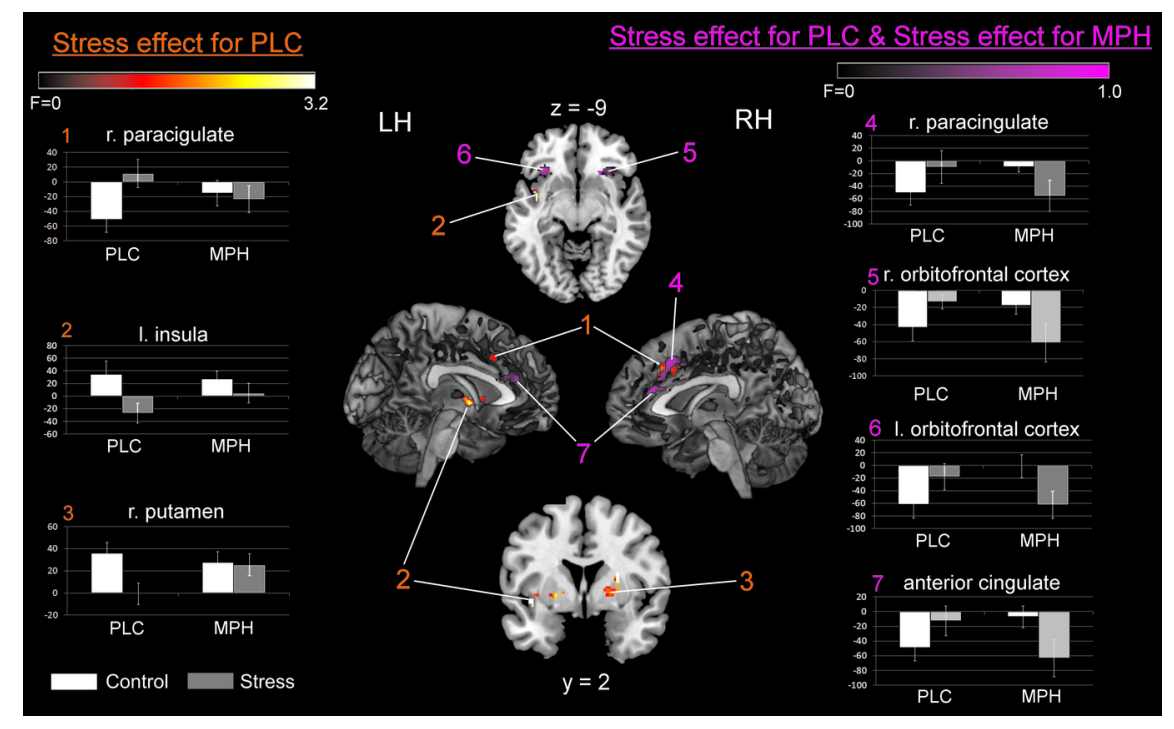

\section{Hosted file}

Table_1.docx available at https://authorea.com/users/302245/articles/432497-dopaminergicmodulation-of-stress-induced-alterations-in-goal-directed-behaviour-and-associatedbrain-activation 